\title{
e-CORE OF DOUBLE SEQUENCES
}

\author{
Y. SEVER ${ }^{1, *}$ and $\ddot{O}$. TALO ${ }^{2}$ \\ ${ }^{1}$ Department of Mathematics, Faculty of Art and Sciences, Afyon Kocatepe University, \\ 03200 Afyonkarahisar, Turkey \\ e-mails: yurdalsever@hotmail.com, ysever@aku.edu.tr \\ ${ }^{2}$ Department of Mathematics, Faculty of Art and Sciences, Celal Bayar University, 45040 Manisa, \\ Turkey \\ e-mails: ozertalo@hotmail.com,ozer.talo@cbu.edu.tr
}

(Received December 12, 2013; revised January 9, 2014; accepted January 13, 2014)

\begin{abstract}
Boos, Leiger and Zeller $[1,2]$ defined the concept of $e$-convergence. In this paper we introduce the concepts of e-limit superior and inferior for real double sequences and prove some fundamental properties of e-limit superior and inferior. In addition to these results we define e-core for double sequences. Also, we show that that if $A$ is a nonnegative $\mathcal{C}_{\mathrm{e}}$-regular matrix then the e-core of $A x$ is contained in e-core of $x$, provided that $A x$ exists.
\end{abstract}

\section{Introduction}

By $\Omega$, we denote the set of all complex valued double sequences, i.e.,

$$
\Omega=\left\{x=\left(x_{m n}\right): x_{m n} \in \mathbb{C} \text { for all } m, n \in \mathbb{N}\right\},
$$

which is a vector space with co-ordinatewise addition and scalar multiplication of double sequences, where $\mathbb{N}$ and $\mathbb{C}$ denote the set of positive integers and the complex field, respectively. Any vector subspace of $\Omega$ is called a double sequence space. The space $\mathcal{M}_{u}$ of all bounded double sequences is defined by

$$
\mathcal{M}_{u}=\left\{x=\left(x_{m n}\right) \in \Omega:\|x\|_{\infty}=\sup _{m, n \in \mathbb{N}}\left|x_{m n}\right|<\infty\right\}
$$

which is a Banach space with the norm $\|\cdot\|_{\infty}$. Consider the sequence $x=\left(x_{m n}\right) \in \Omega$. If for every $\varepsilon>0$ there exists $n_{0}=n_{0}(\varepsilon) \in \mathbb{N}$ such that

\footnotetext{
* Corresponding author.

Key words and phrases: double sequence space, e-convergence, e-limit superior and inferior, core theorem.

Mathematics Subject Classification: primary 40B05, secondary 46A45, 40C05, 40A05.
} 
$\left|x_{m n}-\ell\right|<\varepsilon$ for all $m, n>n_{0}$ then we say that the double sequence $x$ is convergent in the Pringsheim's sense to the limit $\ell$ and write $\mathrm{P}-\lim _{m, n} x_{m n}=\ell$. By $\mathcal{C}_{\mathrm{p}}$, we denote the space of all convergent double sequences in the Pringsheim's sense. It is well-known that there are such sequences in the space $\mathcal{C}_{\mathrm{p}}$ but not in the space $\mathcal{M}_{u}$. So, we may mention the space $\mathcal{C}_{\mathrm{bp}}$ of the double sequences which are both convergent in the Pringsheim's sense and bounded, i.e., $\mathcal{C}_{\mathrm{bp}}=\mathcal{C}_{\mathrm{p}} \cap \mathcal{M}_{u}$. Móricz [8] proved that $\mathcal{C}_{\mathrm{bp}}$ is a Banach space with the norm $\|\cdot\|_{\infty}$. By $\mathcal{C}_{\mathrm{bp} 0}$, we denote the space of double sequences which are both convergent to zero in the Pringsheim's sense and bounded.

Boos, Legier and Zeller [1,2] introduced and investigated the notion of $e$ convergence of double sequences, which is essentially weaker than the Pringsheim convergence. Zeltser [16] characterized SM-methods (see $[12,14]$ ) mapping bounded or convergent sequences into e-, be- or c-convergent double sequences, as well as 4-dimensional matrices being conservative with respect to the one of these notions of convergence. A double sequence $x=\left(x_{k l}\right) \in \Omega$ is said to be $e$-convergent to a number $a$ if

$$
\forall \varepsilon>0 \exists l_{0} \in \mathbb{N}, \forall l \geqq l_{0}, \exists k_{l} \in \mathbb{N} \ni \forall k \geqq k_{l} \Rightarrow\left|x_{k l}-a\right|<\varepsilon .
$$

The space of all double sequences converging in this way is denoted by $\mathcal{C}_{\mathrm{e}}$. More precisely,

$$
\begin{gathered}
\mathcal{C}_{\mathrm{e}}:=\left\{x=\left(x_{k l}\right) \in \Omega \mid \exists a \in \mathbb{C}, \forall \varepsilon>0 \exists l_{0} \in \mathbb{N}, \forall l \geqq l_{0},\right. \\
\left.\exists k_{l} \in \mathbb{N} \ni \forall k \geqq k_{l} \Rightarrow\left|x_{k l}-a\right|<\varepsilon\right\} \\
=\left\{x=\left(x_{k l}\right) \in \Omega\left|\exists a \in \mathbb{C}: \lim _{l} \varlimsup_{k}\right| x_{k l}-a \mid=0\right\} .
\end{gathered}
$$

The subspace

$$
\mathcal{C}_{\text {be }}=\left\{x \in \mathcal{C}_{\mathrm{e}} \mid \forall l \in \mathbb{N}:\left(x_{k l}\right)_{k} \in l_{\infty}\right\}
$$

of $\mathcal{C}_{\mathrm{e}}$, where $l_{\infty}$ is the space of all bounded sequences.

Definition 1.1 [16]. A real double sequence $x=\left(x_{k l}\right)$ is said to be ebounded if $\overline{\lim }_{l} \varlimsup_{k}\left|x_{k l}\right|<\infty$. That is, a real double sequence $x=\left(x_{k l}\right)$ is said to be e-bounded if there exists $M>0$ such that

$$
\exists l_{0} \in \mathbb{N}, \forall l \geqq l_{0}, \exists k_{l} \in \mathbb{N} \ni \forall k \geqq k_{l} \Rightarrow\left|x_{k l}\right|<M \text {. }
$$

Patterson [11] gave the definition of subsequence, the Pringsheim limit inferior and limit superior of double sequences.

Definition 1.2 [11]. Let $x=\left(x_{k l}\right)$ be a double sequence of real numbers and for each $n$, let $\alpha_{n}=\sup _{n}\left\{x_{k l}: k, l \geqq n\right\}$. The Pringsheim limit superior of $x$ is defined as follows: 
(i) if $\alpha_{n}=+\infty$ for each $n$, then P- $\lim \sup x:=+\infty$;

(ii) if $\alpha_{n}<+\infty$ for some $n$, then P- $\lim \sup x:=\inf _{n}\left\{\alpha_{n}\right\}$.

Similarly, let $\beta_{n}=\inf _{n}\left\{x_{k l}: k, l \geqq n\right\}$. Then the Pringsheim limit inferior of $x=\left(x_{k l}\right)$ is defined as follows:

(i) if $\beta_{n}=-\infty$ for each $n$, then $\mathrm{P}$ - liminf $x:=-\infty$;

(ii) if $\beta_{n}>-\infty$ for some $n$, then $\mathrm{P}$ - $\liminf x:=\sup _{n}\left\{\beta_{n}\right\}$.

Definition 1.3. A number $\alpha$ is called an e-limit point of the double sequence $x=\left(x_{k l}\right)$ provided that there exists a subsequence $y=\left(y_{k l}\right)$ of $x=\left(x_{k l}\right)$ that has e-limit $\alpha$ : e- $\lim _{k l} y_{k l}=\alpha$.

ExAMPLE 1.4. The following is an example of $x=\left(x_{k l}\right)$ which is econvergent; however, $x$ is not $\mathrm{P}$-convergent. Define

$$
x_{k l}:= \begin{cases}k, & k=l, \\ 1, & k<l, \\ 0, & k>l .\end{cases}
$$

Then, it is easy to see that e- $\lim _{k l} x_{k l}=0$, whereas $\mathrm{P}-\lim _{k l} x_{k l}$ does not exist.

Let $\lambda$ be the space of double sequences, converging with respect to some linear convergence rule $v$ - $\lim : \lambda \rightarrow \mathbb{C}$. The sum of a double series $\sum_{i, j} x_{i j}$ with respect to this rule is defined by $v-\sum_{i j} x_{i j}=v-\lim _{m, n} \sum_{i=1}^{m} \sum_{j=1}^{n} x_{i j}$. Let $\lambda, \mu$ be two spaces of double sequences, converging with respect to the linear convergence rules $v_{1}$ - lim and $v_{2}$ - lim, respectively, and let $A=\left(a_{m n k l}\right)$ also be a four dimensional matrix of complex numbers. Define the set

$$
\lambda_{A}^{\left(v_{2}\right)}=\left\{\left(x_{k l}\right) \in \Omega: A x=\left(v_{2}-\sum_{k, l} a_{m n k l} x_{k l}\right)_{m, n \in \mathbb{N}} \text { exists and } A x \in \lambda\right\} .
$$

Then, we say, with the notation of (1.1), that $A$ maps the space $\lambda$ into the space $\mu$ if $\mu \subset \lambda_{A}^{\left(v_{2}\right)}$ and denote the set of all four dimensional matrices, mapping the space $\lambda$ into the space $\mu$, by $(\lambda: \mu)$. It is trivial that for any matrix $A \in(\lambda: \mu),\left(a_{m n k l}\right)_{k, l \in \mathbb{N}}$ is in the $\beta\left(v_{2}\right)$-dual $\lambda^{\beta\left(v_{2}\right)}$ of the space $\lambda$ for all $m, n$ $\in \mathbb{N}$. An infinite matrix $A$ is said to be $\mathcal{C}_{v}$-conservative if $\mathcal{C}_{v} \subset\left(\mathcal{C}_{v}\right)_{A}$. For more details on double sequences, 3-dimensional and 4-dimensional matrices, we refer to $[6,13,15-18]$.

We refer the reader to [16] for the basic terminology. Denote by $w$ the vector space of all number sequences

$$
\varphi:=\left\{x \in w: \exists k_{0} \in \mathbb{N} \forall k>k_{0}: x_{k}=0\right\} .
$$


We write $e^{k l}(k, l \in \mathbb{N})$ for the double sequence with

$$
e_{i j}^{k l}:= \begin{cases}1, & \text { if }(k, l)=(i, j), \\ 0, & \text { otherwise }\end{cases}
$$

Let

$$
e=\sum_{k, l} e^{k l}, \quad e^{l}=\sum_{k} e^{k l}(l \in \mathbb{N}) \quad \text { and } \quad e_{k}=\sum_{l} e^{k l}(k \in \mathbb{N})
$$

and $\Phi=\operatorname{span}\left\{e^{k l}: k, l \in \mathbb{N}\right\}$, that is, $\Phi:=\left\{x \in \Omega: \exists k_{0} \in \mathbb{N}: k \geqq k_{0}\right.$ or $\left.l \geqq k_{0} \Rightarrow x_{k l}=0\right\}$.

Theorem 1.5 [16, p. 106]. A 3-dimensional matrix $B=\left(b_{m n k}\right)$ maps $w$ into $C_{\mathrm{e}}$ if and only if the following conditions hold:

(i) $b^{(m, n)}:=\left(b_{m n k}\right)_{k} \in \varphi$ for every $m ; n \in \mathbb{N}$,

(ii) for every $k \in \mathbb{N}$, the limit $b_{k}:=\mathrm{e}-\lim _{m, n} b_{m n k}$ exist,

(iii) there exists $N \in \mathbb{N}$ such that

$$
\forall n \geqq N \exists K(n) \in \mathbb{N}: k>K(n) \Rightarrow b_{m n k}=0 \quad(m \in \mathbb{N}),
$$

(iv) there exist $N, K \in \mathbb{N}$ such that $\lim _{m} b_{m n k}=0 k \geqq K, n \geqq N$.

Under these circumstances, $b:=\left(b_{k}\right) \in \varphi$ and $\mathrm{e}-\lim _{m, n}[B z]_{m n}=\Sigma_{k} b_{k} z_{k}$ $(z \in w)$.

Theorem $1.6\left[16\right.$, p. 110]. (a) A 4-dimensional matrix $A=\left(a_{m n k l}\right)$ is $\mathcal{C}_{\mathrm{e}}$-conservative if and only if the following conditions hold:

(i) $a^{(m, n)}=\left(a_{m n k l}\right)_{k l} \in \Phi$ for every $m, n \in \mathbb{N}$,

(ii) for every $l_{0} \in \mathbb{N}$, the matrix $\left(a_{m n k l}\right)_{m, n, k}$ maps $w$ into $\mathcal{C}_{\mathrm{e}}$,

(iii) the limit $v:=\mathrm{e}-\lim _{m, n} \sum_{k l} a_{m n k l}$ exists,

(iv) there exists $n_{0} \in \mathbb{N}$ such that

$$
\forall n \geqq n_{0} \exists L(n) \in \mathbb{N}: a^{(m, n)}=\sum_{l=1}^{L(n)} a^{(m, n)} e^{l} \quad(m \in \mathbb{N}),
$$
$(k \in \mathbb{N})$,

(v) there exists $L, N \in \mathbb{N}$ such that $l \geqq L, n \geqq N \Rightarrow \lim _{m} a_{m n k l}=0$

(vi) there exists $N^{\prime} \in \mathbb{N}$ and $m_{n}$ such that

$$
\sup _{\substack{n \geqq N^{\prime} \\ m \geqq m_{n}}} \sum_{k l}\left|a_{m n k l}\right|<\infty .
$$


Under these circumstances, $a=\left(a_{k l}\right)=\left(\mathrm{e}-\lim _{m, n} a_{m n k l}\right) \in \Phi$, and

$$
\mathrm{e}-\lim _{m, n}[A x]_{m n}=\sum_{k l} a_{k l} x_{k l}+\left(v-\sum_{k l} a_{k l}\right) \mathrm{e}-\lim _{m, n} x_{m n} \quad\left(x \in \mathcal{C}_{\mathrm{e}}\right) .
$$

(b) $A=\left(a_{m n k l}\right)$ is $\mathcal{C}_{\mathrm{e}}$-regular if and only if the conditions; (i)-(vi) hold with $a_{k l}=0(k, l \in \mathbb{N})$ and $v=1$.

By using the definitions of Pringsheim limit inferior, limit superior and the Pringsheim core of a double sequence with the notion of the regularity of four dimensional matrices, Patterson [11] gave some results on core of double sequences. Mursaleen [9], Mursaleen and Edely [10] defined the almost strong regularity of matrices for double sequences, applied these matrices to establish a core theorem, introduced the $M$-core for double sequences, and determined those four dimensional matrices transforming every bounded double sequence $x=\left(x_{k l}\right)$ into one whose core is a subset of the $M$-core of $x$. Recently, Çakan and Altay [4] investigated statistical core for double sequences and studied an inequality related to the statistical and P-cores of bounded double sequences. Gökhan, Çolak and Mursaleen [5] generalized the Pringsheim core for bounded double sequences and gave some core theorems via matrix classes. Çakan, Altay and Mursaleen [3] introduced $\sigma$-convergence of a double sequence and defined the $\sigma$-core for double sequences and determined a class of four-dimensional matrices such that P-core $(A x) \subset \sigma$-core $(x)$ for all $x \in \mathcal{M}_{u}$. Kumar [7] defined $\mathcal{I}$-limit inferior, $\mathcal{I}$-limit superior and $\mathcal{I}$-core for real double sequences.

In this paper we introduce the concepts of e-limit superior and inferior for real double sequences and prove some fundamental properties of e-limit superior and inferior. In addition to these results we define e-core for double sequences. Also, we show that if $A$ is a nonnegative $\mathcal{C}_{\mathrm{e}}$-regular matrix then the e-core of $A x$ is contained in the e-core of $x$, provided that $A x$ exists.

\section{Main result}

Definition 2.1. Let $x=\left(x_{k l}\right)$ be a double sequence of real numbers. e-limit superior of $x=\left(x_{k l}\right)$ is defined by

$$
\text { e- } \lim \sup x:= \begin{cases}\inf B_{x}, & B_{x} \neq \emptyset \\ \infty, & \text { otherwise. }\end{cases}
$$

and e-limit inferior of $x=\left(x_{k l}\right)$ is defined by

$$
\text { e- } \liminf x:= \begin{cases}\sup A_{x}, & A_{x} \neq \emptyset \\ -\infty, & \text { otherwise }\end{cases}
$$


where

$$
A_{x}:=\left\{a \in \mathbb{R}: \exists l_{0} \in \mathbb{N}, \forall l \geqq l_{0}, \exists k_{l} \in \mathbb{N} \ni \forall k \geqq k_{l}: x_{k l}>a\right\}
$$

and

$$
B_{x}:=\left\{b \in \mathbb{R}: \exists l_{0} \in \mathbb{N}, \forall l \geqq l_{0}, \exists k_{l} \in \mathbb{N} \ni \forall k \geqq k_{l}: x_{k l}<b\right\} .
$$

Clearly, if a real double sequence $x=\left(x_{k l}\right)$ is e-bounded, then $A_{x} \neq \emptyset$ and $B_{x} \neq \emptyset$. Therefore e- $\lim \inf x$ and e- $\lim \sup x$ are both finite numbers.

THEOREM 2.2. Let $x=\left(x_{k l}\right)$ be a double sequence of real numbers. If $u=\mathrm{e}-\lim \sup x$ is finite, then for every $\varepsilon>0 \exists l_{0} \in \mathbb{N}, \forall l \geqq l_{0}, \exists k_{l} \in \mathbb{N} \ni$ $\forall k \geqq k_{l} \Rightarrow x_{k l}<u+\varepsilon$.

Proof. Let e- $\lim \sup x=u$. Then $u=\inf B_{x}$. By the definition of infimum, given $\varepsilon>0$, there exists $u_{\varepsilon} \in B_{x}$ such that $u_{\varepsilon} \leqq u+\varepsilon$. Since $u_{\varepsilon} \in B_{x}$ and taking into consideration the definition of the set $B_{x}, \exists l_{0} \in \mathbb{N}, \forall l \geqq l_{0}$, $\exists k_{l} \in \mathbb{N} \ni \forall k \geqq k_{l}$ we get $x_{k l}<u_{\varepsilon}$. Therefore, for every $\varepsilon>0 \exists l_{0} \in \mathbb{N}$, $\forall l \geqq l_{0}, \exists k_{l} \in \mathbb{N} \ni \forall k \geqq k_{l}$ we obtain that $x_{k l}<u+\varepsilon$.

The proof of the following theorem is the same as above and so we omit it.

THEOREM 2.3. Let $x=\left(x_{k l}\right)$ be a double sequence of real numbers. If $\mathrm{e}-\liminf x=v$ is finite, then given $\varepsilon>0, \exists l_{0} \in \mathbb{N}, \forall l \geqq l_{0}, \exists k_{l} \in \mathbb{N}$ $\ni \forall k \geqq k_{l} \Rightarrow x_{k l}>v-\varepsilon$.

The proof of the following lemma is the same as the proof for convergence in Pringsheim sense and so we omit it.

LEMMA 2.4. For any real-valued double sequence $x$, e- $\lim \sup (-x)=$ $-(\mathrm{e}-\lim \inf x)$

THEOREM 2.5. For any real-valued double sequence $x$, e- $\liminf x \leqq$ $\mathrm{e}-\lim \sup x$.

Proof. If e- $\lim \sup x=-\infty$, then we have $B_{x}=\mathbb{R}$ and $A_{x}=\emptyset$. This implies that e- $\lim \inf x=-\infty$. If e- $\lim \sup x=\infty$, then we have nothing to prove. Assume that e- $\lim \sup x$ is finite. Let $a \in A_{x}$ and $b \in B_{x}$. Thus, we can find $x_{k l}$ such that $a<x_{k l}<b$. That is, any member of $B_{x}$ is greater than all members of $A_{x}$. This completes the proof.

TheOREM 2.6. For any real-valued double sequence $x$,

$$
\mathrm{e}-\lim \sup x=\mathrm{e}-\liminf x=\ell \quad \text { if and only if } \mathrm{e}-\lim x=\ell \text {. }
$$


Proof. Let e- $\lim x=\ell$. Then for any $\varepsilon>0, \exists l_{0} \in \mathbb{N}, \forall l \geqq l_{0}, \exists k_{l} \in \mathbb{N}$ $\ni \forall k \geqq k_{l}$

$$
\ell-\varepsilon<x_{k l}<\ell+\varepsilon,
$$

which implies that $\ell+\varepsilon \in B_{x}$ and $\ell-\varepsilon \in A_{x}$. Thus we obtain

$$
\ell-\varepsilon \leqq \mathrm{e}-\liminf x=\sup A_{x} \leqq \mathrm{e}-\lim \sup x=\inf B_{x} \leqq \ell+\varepsilon .
$$

Since $\varepsilon$ is arbitrary, e- $\lim \sup x=\mathrm{e}-\lim \inf x=\ell$ holds.

On the other hand, let $\mathrm{e}-\lim \sup x=\mathrm{e}-\lim \inf x=\ell$. So, for any $\varepsilon>0$ $\exists l_{1} \in \mathbb{N}, \forall l \geqq l_{1}, \exists k_{l} \in \mathbb{N} \ni \forall k \geqq k_{l} \Rightarrow x_{k l}<\ell+\varepsilon$ and $\exists l_{2} \in \mathbb{N}, \forall l \geqq l_{2}$, $\exists k_{l} \in \mathbb{N} \ni \forall k \geqq k_{l} \Rightarrow x_{k l}>\ell-\varepsilon$. Let $\ell_{0}=\max \left\{\ell_{1}, \ell_{2}\right\}$. Then $\forall l \geqq l_{0}, \exists k_{l}$ $\in \mathbb{N} \ni \forall k \geqq k_{l}$ we get $\ell-\varepsilon<x_{k l}<\ell+\varepsilon$, that is, $\left|x_{k l}-\ell\right|<\varepsilon$. This means that $\mathrm{e}-\lim x=\ell$.

TheOREM 2.7. If $x=\left(x_{k l}\right)$ and $y=\left(y_{k l}\right)$ are two e-bounded real double sequences, then we have:

(i) $\mathrm{e}-\lim \sup (x+y) \leqq \mathrm{e}-\lim \sup x+\mathrm{e}-\lim \sup y$,

(ii) $\mathrm{e}-\liminf (x+y) \geqq \mathrm{e}-\liminf x+\mathrm{e}-\lim \inf y$.

Proof. (i) Since $x=\left(x_{k l}\right)$ and $y=\left(y_{k l}\right)$ are e-bounded real double sequences, e- $\lim \sup x$ and e- $\lim \sup y$ are both finite. Suppose that e- $\lim \sup x=\alpha, \mathrm{e}-\lim \sup y=\beta$ and

$$
\begin{aligned}
& B_{(x+y)}:=\left\{b \in \mathbb{R}: \exists l_{0} \in \mathbb{N}, \forall l \geqq l_{0}, \exists k_{l} \in \mathbb{N} \ni \forall k \geqq k_{l} \Rightarrow\right. \\
&\left.x_{k l}+y_{k l}<b\right\} .
\end{aligned}
$$

For given $\varepsilon>0$,

$$
\exists l_{1} \in \mathbb{N}, \forall l \geqq l_{1}, \exists k_{l} \in \mathbb{N} \ni \forall k \geqq k_{l} \Rightarrow x_{k l}<\alpha+\varepsilon / 2
$$

and

$$
\exists l_{2} \in \mathbb{N}, \forall l \geqq l_{2}, \exists k_{l} \in \mathbb{N} \ni \forall k \geqq k_{l} \Rightarrow y_{k l}<\beta+\varepsilon / 2 .
$$

Let $l_{0}=\max \left\{l_{1}, l_{2}\right\}$. Then

$$
\forall l \geqq l_{0}, \exists k_{l} \in \mathbb{N} \ni \forall k \geqq k_{l} \Rightarrow x_{k l}+y_{k l}<\alpha+\beta+\varepsilon .
$$

Therefore we get $\alpha+\beta+\varepsilon \in B_{(x+y)}$. So,

$$
\text { e- } \lim \sup (x+y)=\inf B_{(x+y)} \leqq \alpha+\beta+\varepsilon .
$$

Since $\varepsilon$ is arbitrary, we obtain

$$
\text { e- } \lim \sup (x+y) \leqq \mathrm{e}-\lim \sup x+\mathrm{e}-\lim \sup y .
$$

(ii) It can be proved by the same way as above. 
Theorem 2.8. P- $\lim \inf x \leqq \mathrm{e}-\lim \inf x \leqq \mathrm{e}-\lim \sup x \leqq \mathrm{P}-\lim \sup x$.

Proof. Let P- $\lim \sup x=\alpha$. Since $\alpha=\inf _{n} \sup _{k, l \geqq n} x_{k l}$, given $\varepsilon>0$ there exists $n_{\varepsilon}$ such that

$$
\sup _{k, l \geqq n_{\varepsilon}} x_{k l}<\alpha+\varepsilon
$$

Hence for all $k, l \geqq n_{\varepsilon}$ we get $x_{k l}<\alpha+\varepsilon$. Therefore $l \geqq l_{0}=n_{\varepsilon}, \exists k_{l}=n_{\varepsilon}$ $\in \mathbb{N} \ni \forall k \geqq k_{l} \Rightarrow x_{k l}<\alpha+\varepsilon$. This means that $\alpha+\varepsilon \in B_{x}$. So

$$
\text { e- } \lim \sup x=\inf B_{x} \leqq \alpha+\varepsilon .
$$

Hence $\varepsilon$ is arbitrary and we obtain e- $\lim \sup x \leqq \alpha$. Similarly, it can be shown that $\mathrm{P}-\lim \inf x \leqq \mathrm{e}-\lim \inf x$.

EXAMPLE 2.9. The following is an example of a sequence $x=\left(x_{k l}\right)$ which has finite e- limsup and e- liminf; however, P-limsup and P-lim inf are not finite. Define

$$
x_{k l}:= \begin{cases}k, & k=l \\ -k, & k=l+1 \\ 1, & k<l+1 \text { and } k+l \text { is even, } \\ -1, & k<l+1 \text { and } k+l \text { is odd } \\ 0, & k>l .\end{cases}
$$

Then, it is easy to see that $A_{x}=(-\infty,-1)$ and $B_{x}=(1,+\infty)$. So, e- $\lim \sup _{k l} x_{k l}=1$ and e- $\liminf \operatorname{ing}_{k l} x_{k l}=-1$ but P-limsup $\sup _{k l} x_{k l}=+\infty$ and $\mathrm{P}-\liminf \operatorname{il}_{k l} x_{k l}=-\infty$.

In analogy to the $P$-core [11], statistical core [4] and $\mathcal{I}$-core [7] we define the e-core of double sequences as follows.

Definition 2.10. For any e-bounded real double sequence $x=\left(x_{k l}\right)$, the e-core of $x$ is defined as the closed interval $[\mathrm{e}-\lim \inf x, \mathrm{e}-\lim \sup x$ ]. In case $x$ is not e-bounded, e-core of the sequence $x$ is defined by either $(-\infty, \mathrm{e}-\lim \sup x],[\mathrm{e}-\lim \inf x, \infty)$ or $(-\infty, \infty)$. e-core $(x)$ will denote e-core of the sequence $x=\left(x_{k l}\right)$.

From Theorem 2.8 , it is clear that $\mathrm{e}-\operatorname{core}(x) \subset P$-core $(x)$, for any real double sequence $x$.

TheOREM 2.11. Let $x=\left(x_{k l}\right), y=\left(y_{k l}\right)$ be e-bounded double sequences. If $\mathrm{e}-\lim _{k l}\left|x_{k l}-y_{k l}\right|=0$, then $\mathrm{e}-\operatorname{core}(x)=\mathrm{e}-\operatorname{core}(y)$. 
Proof. Suppose that e- $\lim \sup x=\alpha$, e- $\lim \sup y=\beta$ and e- $\lim _{k l} \mid x_{k l}-$ $y_{k l} \mid=0$. Then, for each $\varepsilon>0$

$$
\begin{gathered}
\exists l_{1} \in \mathbb{N}, \forall l \geqq l_{1}, \exists k_{l} \in \mathbb{N} \ni \forall k \geqq k_{l} \Rightarrow y_{k l}-\varepsilon / 2<x_{k l}<y_{k l}+\varepsilon / 2, \\
\exists l_{2} \in \mathbb{N}, \forall l \geqq l_{2}, \exists k_{l} \in \mathbb{N} \ni \forall k \geqq k_{l} \Rightarrow y_{k l}<\beta+\varepsilon / 2
\end{gathered}
$$

and

$$
\exists l_{3} \in \mathbb{N}, \forall l \geqq l_{3}, \exists k_{l} \in \mathbb{N} \ni \forall k \geqq k_{l} \Rightarrow x_{k l}<\alpha+\varepsilon / 2 .
$$

Let $l_{0}=\max \left\{l_{1}, l_{2}, l_{3}\right\}$. Then

$$
\forall l \geqq l_{0}, \exists k_{l} \in \mathbb{N} \ni \forall k \geqq k_{l} \Rightarrow x_{k l}<\beta+\varepsilon
$$

and

$$
\forall l \geqq l_{0}, \exists k_{l} \in \mathbb{N} \ni \forall k \geqq k_{l} \Rightarrow y_{k l}<\alpha+\varepsilon .
$$

Therefore we get $\beta+\varepsilon \in B_{x}$ and $\alpha+\varepsilon \in B_{y}$. So, $\alpha=\inf B_{x} \leqq \beta+\varepsilon$ and $\beta=\inf B_{y} \leqq \alpha+\varepsilon$. Since $\varepsilon$ is arbitrary, we obtain $\alpha \leqq \beta$ and $\beta \leqq \alpha$. This means that $\alpha=\beta$. Similarly, it can be shown that e- $\lim \inf x=\mathrm{e}-\lim \inf y$. Therefore, $\mathrm{e}-\operatorname{core}(x)=\mathrm{e}-\operatorname{core}(y)$.

Theorem 2.12. Let $A$ be a 4-dimensional $\mathcal{C}_{\mathrm{e}}$-regular matrix with positive real entries. Then,

$$
\text { e- } \lim \sup A x \leqq \mathrm{e}-\lim \sup x
$$

for all real-valued bounded double sequences $x=\left(x_{k l}\right)$.

Proof. Let $x=\left(x_{k l}\right)$ be a double bounded sequence and let $A$ be a $\mathcal{C}_{\mathrm{e}}$-regular summability matrix. We need to show that e- $\lim \sup (A x)$ $\leqq \mathrm{e}-\lim \sup x$. Suppose that $\mathrm{e}-\lim \sup x=\ell$. So, for any $\varepsilon>0 \exists P_{1} \in \mathbb{N}$, $\bar{\forall} l \geqq P_{1}, \exists k_{l} \in \mathbb{N} \ni \forall k \geqq k_{l} \Rightarrow x_{k l}<\ell+\varepsilon$.

$$
\begin{gathered}
\sum_{k, l=1,1}^{\infty, \infty} a_{m n k l} x_{k l}=\sum_{l=P_{1}}^{\infty} \sum_{k=k_{l}}^{\infty} a_{m n k l} x_{k l} \\
+\sum_{l=P_{1}}^{\infty} \sum_{k=1}^{k=k_{l}-1} a_{m n k l} x_{k l}+\sum_{l=1}^{l=P_{1}-1} \sum_{k=1}^{\infty} a_{m n k l} x_{k l} \\
\leqq(\ell+\varepsilon) \sum_{l=P_{1}}^{\infty} \sum_{k=k_{l}}^{\infty} a_{m n k l}+\|x\| \sum_{l=P_{1}}^{\infty} \sum_{k=1}^{k=k_{l}-1} a_{m n k l}+\|x\| \sum_{l=1}^{l=P_{1}-1} \sum_{k=1}^{\infty} a_{m n k l}
\end{gathered}
$$


Taking into account the condition of $e$-regularity and taking e- limsup of both side, we get

$$
\text { e- } \lim \sup A x \leqq l+\varepsilon .
$$

Since $\varepsilon$ is arbitrary, we have (2.2).

From Theorem 2.12 and Lemma 2.4 we obtain the following result.

Corollary 2.13. Let $A$ be a 4-dimensional $\mathcal{C}_{\mathrm{e}}$-regular matrix with positive real entries. Then,

$$
\mathrm{e}-\operatorname{core}(A x) \subset \mathrm{e}-\operatorname{core}(x)
$$

for all real-valued bounded double sequences $x=\left(x_{k l}\right)$.

Acknowledgement. We have benefited a lot from discussions with Professor Bilâl Altay about this work. We express our gratitude for his valuable helps. The authors thank the referee for thoroughly reading the paper and for providing valuable comments and useful suggestions for the improvement of this paper.

\section{References}

[1] J. Boos, T. Leiger and K. Zeller, Consistency theory for SM-methods, Acta Math. Hungar., 76 (1997), 83-116.

[2] J. Boos, Classical and Modern Methods in Summability, Oxford University Press Inc. (New York, 2000).

[3] C. Çakan, B. Altay and M. Mursaleen, The $\sigma$-convergence and $\sigma$-core of double sequences, Appl. Math. Lett., 19 (2006), 1122-1128.

[4] C. Çakan and B. Altay, Statistically boundedness and statistical core of double sequences, J. Math. Anal. Appl., 317 (2006), 690-697.

[5] A. Gökhan, R. Çolak and M. Mursaleen, Some matrix transformations and generalized core of double sequences, Math. Comput. Modelling, 49 (2009), 1721-1731.

[6] H. J. Hamilton, Transformations of multiple of sequences, Duke Math. J., 2 (1936), $29-60$.

[7] V. Kumar, I-core of double sequences, Int. J. Contemp. Math. Sciences, 23 (2007), $1137-1145$.

[8] F. Móricz, Extensions of the spaces $c$ and $c_{0}$ from single to double sequences, Acta Math. Hungar., 57 (1991), 129-136.

[9] M. Mursaleen, Almost strongly regular matrices and a core theorem for double sequences, J. Math. Anal. Appl., 293 (2004), 523-531.

[10] M. Mursaleen and O. H. H. Edely, Almost convergence and a core theorem for double sequences, J. Math. Anal. Appl., 293 (2004), 532-540.

[11] R. F. Patterson, Double sequence core teorems, Int. J. Math. Math. Sci., 22 (1999), $785-793$.

[12] B. Przybylski, On the perfectness of methods defined by the iteration product of matrix transformations, Doctorial Thesis, University of Lódź (1977).

[13] G. M. Robison, Divergent double sequences and series, Trans. Amer. Math. Soc., 28 (1926), 50-73. 
[14] K. Zeller und W. Beekmann, Theorie der Limitierungsverfahren (2. Aufl.), Springer (Berlin - Heidelberg - New York, 1970).

[15] M. Zeltser, Matrix transformations of double sequences, Acta Comment. Univ. Tartu Math., 4 (2000), 39-51.

[16] M. Zeltser, Investigation of Double Sequence Spaces by Soft and Hard Analitical Methods, Dissertationes Mathematicae Universitatis Tartuensis 25, Tartu University Press, Univ. of Tartu, Faculty of Mathematics and Computer Science, (Tartu, 2001).

[17] M. Zeltser, On conservative and coercive SM-methods, Proc. Est. Acad. Sci. Phys. Math., 50 (2001), 76-85.

[18] M. Zeltser, On conservative matrix methods for double sequence spaces, Acta Math. Hungar., 95 (2002), 225-242. 